\title{
ON COHOMOLOGY OF LIE ALGEBRAS
}

GEORGE F. LEGER, JR.

Let $G$ be a Lie algebra, $L$ an ideal of $G$ and $M$ a $G$-module. The $n$-dimensional cochains for $L$ in $M$ are $n$-linear alternating functions on $L^{n}$ with values in $M$. These cochains form a vector space $C^{n}(L, M)$ over the ground field of $L$; we identify $C^{0}(L, M)$ with $M . C^{0}(L, M)$ is already a $G$-module and for $n>0, f$ in $C^{n}(L, M)$ and $\gamma$ in $G$ put $(\gamma \cdot f)\left(\sigma_{1}, \cdots, \sigma_{n}\right)$

$$
=\gamma \cdot f\left(\sigma_{1}, \cdots, \sigma_{n}\right)-\sum_{i=1}^{n} f\left(\sigma_{1}, \cdots, \sigma_{i-1},\left[\gamma, \sigma_{i}\right], \sigma_{i+1}, \cdots, \sigma_{n}\right)
$$

for all $\sigma_{1}, \cdots, \sigma_{n}$ in $L$.

This definition makes $C^{n}(L, M)$ into a $G$-module and further $\gamma \cdot(\delta f)=\delta(\gamma \cdot f)$ where $\delta$ is the coboundary operator: $C^{n}(L, M)$ $\rightarrow C^{n+1}(L, M)$ so that the cohomology groups $H^{n}(L, M)$ take on the structure of a $G$-module. Professor Hochschild suggested to the author that he define an operation of $G$ on the standard interpretations of the low dimensional cohomology groups which would agree with the operation of $G$ on $H^{n}(L, M)$ as given above.

This problem has an analogue in the cohomology of groups. There, if $L$ is a normal subgroup of a group $G$ and $f$ is any function of $L^{n+1}$ to $M$, the operation

$$
(\gamma \cdot f)\left(\sigma_{0}, \cdots, \sigma_{n}\right)=\gamma \cdot f\left(\gamma^{-1} \sigma_{0} \gamma, \cdots, \gamma^{-1} \sigma_{n} \gamma\right)
$$

for $\gamma$ in $G$ and $\sigma_{0}, \cdots, \sigma_{n}$ in $L$, again induces the structure of a $G$-module on the cohomology groups $H^{n}(L, M)$. In an appendix we outline briefly a way of making the standard interpretations of the low dimensional cohomology groups into $G$-modules in a way consistent with the operation of $G$ on the cohomology groups.

The author is pleased to acknowledge his indebtedness to Professor Hochschild for his suggestions and encouragement.

1. Let $G$ be a Lie algebra over a ground field of characteristic 0 , $L$ an ideal of $G$ such that $G / L$ is semi-simple and let $M$ be a $G$-module. We denote by $\rho_{n}$ the restriction homomorphism of $H^{n}(G, M)$ into $H^{n}(L, M)$. For any $G$-module $U$, let $U^{G}=\{u \mid \gamma \cdot u=0$ for all $\gamma$ in $G\}$. A special case of a theorem of Hochschild-Serre [1, p. 603, Theorem $13]^{1}$ is

Presented to the Society, April 21, 1956 and December 29, 1956; received by the editors May 15, 1956 and, in revised form, March 2, 1957.

${ }^{1}$ Numbers in square brackets refer to the bibliography at the end of the paper. 
1.1. TheOREM. If $G / L$ is semi-simple then $\rho_{n}$ is an isomorphism of $H^{n}(G, M)$ onto $H^{n}(L, M)^{G}$ for $n=0,1,2$.

Hochschild-Serre's proof of their Theorem 13 depends on the theory of spectral sequences, and it seems desirable to sketch a direct proof of 1.1 here. Accordingly let $G=K+L$ be a Levi decomposition of $G$.

(1.2) $H^{n}(L, M)^{G}=H^{n}(L, M)^{K}$ for all $n \geqq 0$.

(1.3) Every element of $H^{n}(L, M)^{K}$ has a representative cocycle in $C^{n}(L, M)^{K}$.

$(1.4)^{2}$ If $d f \in C^{n}(L, M)^{K}$ for $f$ in $C^{n-1}(L, M)$ then $\exists g$ in $C^{n-1}(L, M)^{K}$ $\exists d f=d g$.

(1.5) Let $f$ be in $Z^{n}(G, M)$ where $n=1$ or 2 then there exists $f^{*}$ in $Z^{n}(G, M) \ni f^{*}$ is cohomologous to $f$ and

(a) $f^{*}(k)=0$ for all $k$ in $K$ (if $n=1$ ),

(b) $f^{*}\left(\gamma_{1}, \gamma_{2}\right)=0$ if $\gamma_{1}$ or $\gamma_{2}$ is in $K$ (if $n=2$ ).

Proof. (a) If $f \in Z^{1}(G, M)$ then $\left.f\right|_{K} \in Z^{1}(K, M)$ whence $\exists m$ in $C^{0}(K, M)=M \ni f(k)=k \cdot m$ for all $k$ in $K$. Put

$$
f^{*}(\gamma)=(f-d m)(\gamma) \text { for all } \gamma \text { in } G .
$$

(b) If $f \in Z^{2}(G, M)$, define a Lie algebra extension $(E, \pi)$ of $M$ by $G$ as follows: $E=(G, M)$ (direct product) as a vector space with $\left[\left(\gamma_{1}, m_{1}\right),\left(\gamma_{2}, m_{2}\right)\right]=\left(\left[\gamma_{1}, \gamma_{2}\right], \gamma_{1} \cdot m_{2}-\gamma_{2} \cdot m_{1}+f\left(\gamma_{1}, \gamma_{2}\right)\right)$ for all $\gamma_{1}, \gamma_{2}$ in in $G, m_{1}, m_{2}$ in $M ; \pi$ the projection of $E$ onto $G$.

Now $G=K+L$ with $K$ semi-simple. $\pi^{-1}(L)$ is an ideal of $E$ containing $M$ and $E / \pi^{-1}(L) \cong K$. Thus we can find a linear map $\sigma: G \rightarrow E \ni \pi \sigma(\gamma)=\gamma$ for all $\gamma$ in $G$ and $\left.\sigma\right|_{K}$ is a Lie algebra isomorphism of $K$ into $E$. Consider $\pi^{-1}(L)$ and $M$ as $\sigma(K)$-modules in the regular representation. Then since $\sigma(K)$ is semi-simple, there exists a submodule $H$ of $\pi^{-1}(L) \ni \pi^{-1}(L)=H+M$ and $H \cap M=(0) .\left.\pi\right|_{H}$ is a vector space isomorphism of $H$ onto $L$ so we see that we can choose the linear map $\sigma$ so that

(1) $\sigma\left(k_{1}, k_{2}\right]=\left[\sigma\left(k_{1}\right), \sigma\left(k_{2}\right)\right]$ for all $k_{1}, k_{2}$ in $K$.

(2) $E=\sigma(K)+\sigma(L)+M$ direct sum as $\sigma(K)$-modules. Now note that

$$
[\sigma(k), \sigma(x)]-\sigma[k, x] \in \sigma(L) \rightarrow M=(0) \text { for all } k \text { in } K, x \text { in } L .
$$

The cocycle $f^{*} \ni f^{*}\left(\gamma_{1}, \gamma_{2}\right)=\left[\sigma\left(\gamma_{1}\right), \sigma\left(\gamma_{2}\right)\right]-\sigma\left[\gamma_{1}, \gamma_{2}\right]$ is cohomologous to $f$ and has the desired properties.

ProOf of 1.1. If $n=0$ this is trivial. If $n=1$ or 2 and $h \in H^{n}(G, M)$, choose a representative cocycle $f$ of $h$ as in (1.5). It is easy to see that $\left.k \cdot f\right|_{L}=0$ for all $k$ in $K$ where $\left.f\right|_{L}$ is the restriction of $f$ to $L$ so that

$2(1.2),(1.3)$ and (1.4) are proved directly in $[1$, p. 603]. 
$\rho_{n}\left(H^{n}(G, M)\right) \subseteq H^{n}(L, M)^{G}$. Using (1.4) one sees quickly that $\rho_{n}$ is an isomorphism.

To see that $\rho_{n}$ is onto, take $\bar{h}$ in $H^{n}(L, M)^{G}(n=1,2)$ and choose a representative $f$ of $\bar{h}$ in $Z^{n}(L, M)^{K}$ by (1.2) and (1.3). Extend $f$ to $\hat{f}$ in $Z^{n}(G, M)$ by

$$
\begin{array}{lll}
n=1 & \hat{f}(k+x)=f(x) & \text { for all } x \text { in } L, k \text { in } K ;
\end{array} \quad \begin{array}{ll}
\hat{f}(k, \gamma)=0 & \text { for all } \gamma \text { in } G, k \text { in } K, \\
\hat{f}\left(x_{1}, x_{2}\right)=f\left(x_{1}, x_{2}\right) & \text { for all } x_{1}, x_{2} \text { in } L
\end{array}
$$

then $\rho_{n}\left(\hat{f}+d C^{n-1}(G, M)\right)=\bar{h}$.

1.6. Definition. Let $G$ be a Lie algebra and $V$ an ideal of $G$. If there exists a subalgebra $U$ of $G$ such that $G=U+V$ and $U \cap V=(0)$ we say that $G$ splits over $V$.

As a corollary of 1.2 we have

1.7. Let $G$ be a Lie algebra over a ground field of characteristic 0 and let $L \geqq M$ be ideals of $G$ such that $G / L$ is semi-simple, $M$ is abelian and $L$ splits over $M$. Then $G$ splits over $M$.

It would be interesting to know if the hypothesis that $M$ be abelian can be removed from 1.7.

2. Module extensions. Let $P$ and $Q$ be $G$-modules, $L$ any ideal of $G$. (We drop the requirement that $G / L$ be semi-simple and that the ground field have characteristic 0 .) If $P \subseteq E$ and if the sequence $0 \rightarrow P \rightarrow^{i} E \rightarrow{ }^{\phi} Q \rightarrow 0$ of $L$-modules is exact we say that the pair $(E, \phi)$ is an $L$-extension of $P$ by $Q$. Given two $L$-extensions $\left(E_{1}, \phi_{1}\right)$ and $\left(E_{2}, \phi_{2}\right)$ of $P$ by $Q$, they are called $L$-equivalent if there is an $L$-isomorphism $\tau$ of $E_{1}$ onto $E_{2}$ such that $\tau(p)=p$ for all $p$ in $P$ and such that $\phi_{2} \tau=\phi_{1}$. We denote by $\{(E, \phi)\}$ the class of extensions $L$ equivalent to $(E, \phi)$ and recall that these classes form a vector space, over the ground field of $L$, which we denote by $\operatorname{Ext}_{L}[P, Q]$.

Let $M$ be the vector space of linear maps of $Q$ into $P$ made into a $G$ (and thus also an $L$ )-module by setting

$$
(\gamma \cdot f)(q)=\gamma \cdot f(q)-f(\gamma \cdot q) \text { for all } \gamma \text { in } G, f \text { in } M
$$

and $q$ in $Q$. It is known that $\operatorname{Ext}_{L}[P, Q] \cong H^{1}(L, M)$ as a vector space $\left[2\right.$, p. 562] and we now define an operation of $G$ on $\operatorname{Ext}_{L}[P, Q]$ which agrees with the operation of $G$ on $H^{1}(L, M)$ in the sense that this vector space isomorphism becomes a $G$-isomorphism. Take $\gamma$ in $G$, and $(E, \phi)$ an $L$-module extension of $P$ by $Q$. As a vector space, put

$$
E_{\gamma}^{*}=\left\{\left(e_{1}, e_{2}\right) \mid e_{1}, e_{2} \text { in } E \text { and } \gamma \cdot \phi\left(e_{1}\right)=\phi\left(e_{2}\right)\right\} .
$$


Make $E_{\gamma}^{*}$ into an $L$-module such that

$$
x \cdot\left(e_{1}, e_{2}\right)=\left(x \cdot e_{1}, x \cdot e_{2}+[\gamma, x] \cdot e_{1}\right)
$$

for $x$ in $L$ and

$\left(e_{1}, e_{2}\right)$ in $E_{\gamma}^{*}$. Define a map $\phi_{\gamma}^{*}$ of $E_{\gamma}^{*}$ onto $Q$ by $\phi_{\gamma}^{*}\left(e_{1}, e_{2}\right)=\phi\left(e_{1}\right)$. The kernel of $\phi_{\gamma}^{*}$ is $(P, P)$ and the map $w_{\gamma}:(P, P) \rightarrow P$ such that

$$
w_{\gamma}\left(p_{1}, p_{2}\right)=\gamma \cdot p_{1}-p_{2}
$$

is an $L$-homomorphism of $(P, P)$ onto $P$. The kernel $N_{\gamma}$ of $w_{\gamma}$ consists of all pairs $\left(p_{1}, p_{2}\right)$ for which $p_{2}=\gamma \cdot p_{1} . N_{\gamma}$ is an $L$-submodule of $E_{\gamma}^{*}$ so that we may use $w_{\gamma}$ to induce an $L$-identification of $(P, P) / N_{\gamma}$ with $P$. Finally we put

$$
\gamma \cdot(E, \phi)=\left(E_{\gamma}^{*} / N_{\gamma}, \phi_{\gamma}^{*}\right)
$$

[ $\phi_{\gamma}^{*}$ is used again to denote the $L$-homomorphism of $E_{\gamma}^{*} / N_{\gamma}$ induced by the original $\phi_{\gamma}^{*}$.]

Suppose now that $\left(E_{1}, \phi_{1}\right)$ is an extension of $P$ by $Q$ which is $L$ equivalent to $(E, \phi)$ and let $\psi$ be an $L$-isomorphism of $E$ onto $E_{1}$. The map $\widehat{\psi}: E_{\gamma}^{*} \rightarrow E_{1 \gamma}^{*}$ such that

$$
\widehat{\psi}\left(e_{1}, e_{2}\right)=\left(\psi\left(e_{1}\right), \psi\left(e_{2}\right)\right)
$$

is an $L$-homomorphism which takes $N_{\gamma}$ onto $N_{1 \gamma}$ and induces an $L$ isomorphism of $E_{\gamma}^{*} / N_{\gamma}$ onto $E_{1 \gamma}^{*} / N_{1 \gamma}$. Thus we may put

$$
\gamma\{(E, \phi)\}=\{\gamma \cdot(E, \phi)\} .
$$

2.1. Proposition. If $h$ is the element of $H^{1}(L, M)$ determined by $(E, \phi)$ then the element of $H^{1}(L, M)$ determined by $\gamma \cdot(E, \phi)$ is $\gamma \cdot h$ $[M=$ linear maps of $Q$ into $P]$.

Proof. Let $\psi$ be a linear map of $Q$ into $E$ such that $\phi \psi(q)=q$ for all $q$ in $Q$. Then we know that the $f$ in $C^{1}(L, M)$ such that

$$
f(x)\{q\}=x \cdot \psi(q)-\psi(x \cdot q) \quad \text { for all } x \text { in } L
$$

is an element of $Z^{1}(L, M)$ which represents $h$. Define a linear map $\psi^{*}$ of $Q$ into $E_{\gamma}^{*} / N_{\gamma}$ by

$$
\psi^{*}(q)=(\psi(q), \psi(\gamma \cdot q))+N_{\boldsymbol{\gamma}} .
$$

Then $\phi_{\gamma}^{*} \psi^{*}(q)=q$ for all $q$ in $Q$ and

$$
x \cdot \psi^{*}(q)-\psi^{*}(x \cdot q)=(\gamma \cdot f)(x)\{q\}
$$

for all $x$ in $L$.

2.2. Corollary. The operation of $G$ on $\operatorname{Ext}_{L}[P, Q]$ induces the structure of $a G$-module on $\operatorname{Ext}_{L}[P, Q]$ and the vector space isomorphism 
of $\operatorname{Ext}_{L}[P, Q]$ onto $H^{1}(L, M)$ becomes a G-isomorphism.

Now define a map $\rho: \operatorname{Ext}_{G}[P, Q] \rightarrow \operatorname{Ext}_{L}[P, Q]$ as follows. If $(E, \phi)$ is a $G$-extension of $P$ by $Q$, define an $L$-extension $(\bar{E}, \bar{\phi})$ of $P$ by $Q$ by restricting the operators on $E$ to elements of $L$. As an $L$ module, $\bar{E} \equiv E$ and as an $L$-homomorphism $\bar{\phi} \equiv \phi$. It is evident that if $h$ is the element of $H^{1}(G, M)$ determined by $\{(E, \phi)\}$ then $\rho_{1}(h)$ is the element of $H^{\prime}(L, M)$ determined by $\rho(\{(E, \phi)\})$ where $\rho_{1}$ is the restriction homomorphism. Thus we have:

2.3. Proposition. $\rho\left(\operatorname{Ext}_{G}[P, Q]\right) \subseteq \operatorname{Ext}_{L}[P, Q]^{G}$.

Now we have the diagram:

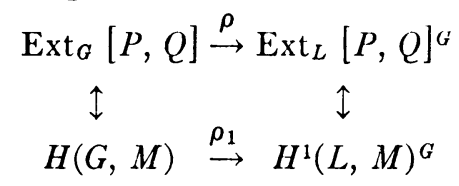

where $\rho$ and $\rho_{1}$ are homomorphisms into. Further this diagram is commutative.

It is easy to find examples showing that $\rho_{1}$ (thus also $\rho$ ) need not be an isomorphism in general. Moreover, the following example (due to Hochschild) shows that $\rho_{1}$ need not even be onto in general: $G$ is 3 dimensional, with basis $x, y, z$ such that $[x, y]=z$ and $z$ is central. Put $L=(z), M$ a trivial 1 dimensional $G$-module. Then every 1 cocycle for $G$ in $M$ must map $L$ into (0); but the map $f$, where $f(a z)$ $=a$, represents a nonzero element of $H^{1}(L, M)^{G}$.

In fact it is an easy consequence of 1.1 and Hochschild-Serre's Theorem $6[1$, p. 596] that

2.4. Proposition. If the ground field of $G$ has characteristic 0 then $\rho_{1}: H^{1}(G, M) \rightarrow H(L, M)^{G}$ is an isomorphism for all G-modules $M$ if and only if $G / L$ is semi-simple.

3. Lie algebra extensions. Let $L$ be an ideal of $G$ and $M$ a $G$-module which we consider as an abelian Lie algebra. An extension of $M$ by $L$ is a pair $(U, \pi)$ such that $M$ is an ideal of $U$ and such that the sequence $0 \rightarrow M \rightarrow{ }^{i} U \rightarrow{ }^{\pi} L \rightarrow 0$ is exact. Two such extensions $\left(U_{1}, \pi_{1}\right)$ and $\left(U_{2}, \pi_{2}\right)$ are called equivalent if there is an isomorphism $\sigma: U_{1} \rightarrow U_{2}$ such that $\sigma(m)=m$ for all $m$ in $M$ and $\pi_{1}=\pi_{2} \sigma$. Using a procedure analogous to the Baer product, one can define directly the structure of a vector space on the classes of extensions $\{(U, \pi)\}$. We denote this vector space by $\operatorname{Ext}(M, L)$. Further it is known, [2, p. 566], that this vector space is isomorphic to $H^{2}(L, M)$.

We define an operation of $G$ on $\operatorname{Ext}(L, M)$ as follows: take $\gamma$ in 
$G$ and $(U, \pi)$ an extension of $M$ by $L$. Put

$$
U_{\gamma}=\left\{\left\langle u_{1}, u_{2}\right\rangle \mid u_{1}, u_{2} \text { in } U,\left[\gamma, \pi\left(u_{1}\right)\right]=\pi\left(u_{2}\right)\right\}
$$

as a vector space and make $U_{\gamma}$ into a Lie algebra by setting

$$
\left[\left\langle u_{1}, u_{2}\right\rangle,\left\langle u_{1}^{\prime}, u_{2}^{\prime}\right\rangle\right]=\left\langle\left[u_{1}, u_{2}^{\prime}\right],\left[u_{1}, u_{2}^{\prime}\right]+\left[u_{2}, u_{1}^{\prime}\right]\right\rangle
$$

for all $\left\langle u_{1}, u_{2}\right\rangle$ and $\left\langle u_{1}^{\prime}, u_{2}^{\prime}\right\rangle$ in $U_{\gamma}$. Define a homomorphism $\pi_{\gamma}$ of $U_{\gamma}$ onto $L$ by $\pi_{\gamma}\left\langle u_{1}, u_{2}\right\rangle=\pi\left(u_{1}\right)$. The kernel of $\pi_{\gamma}$ is $\langle M, M\rangle$. This situation makes $\langle M, M\rangle$ into an $L$-module with $L$ operating on $\langle M, M\rangle$ via $U_{\gamma}$. The map $\omega_{\gamma}:\langle M, M\rangle \rightarrow M$ such that

$$
\omega_{\gamma}\left\langle m_{1}, m_{2}\right\rangle=\gamma \cdot m_{1}-m_{2}
$$

is an $L$-homomorphism of $\langle M, M\rangle$ onto $M$. The kernel $M_{\gamma}$ of $\omega_{\gamma}$ consists of pairs $\langle m, \gamma \cdot m\rangle$ and $M_{\gamma}$ is an ideal of $U_{\gamma}$. Thus $\pi_{\gamma}$ induces a homomorphism (still denoted by $\pi_{\gamma}$ ) of $U_{\gamma} / M_{\gamma}$ onto $L$ with kernel $\langle M, M\rangle / M_{\gamma}$ which we identify with $M$ using $\omega_{\gamma}$. Now define

$$
\gamma \cdot(U, \pi)=\left(U^{\gamma} / M_{\gamma}, \pi_{\gamma}\right) .
$$

If $\left(U_{1}, \pi_{1}\right)$ is equivalent to $(U, \pi)$ under the isomorphism $\psi$, the map $\psi^{*}$ such that $\psi^{*}-\left\langle u_{1}, u_{2}\right\rangle=\left\langle\psi\left(u_{1}\right), \psi\left(u_{2}\right)\right\rangle$ induces an equivalence isomorphism of $\left(U_{\gamma} / M_{\gamma}, \pi_{\gamma}\right)$ with $\left(U_{1 \gamma} / M_{1 \gamma}, \pi_{1 \gamma}\right)$ so that we may put

$$
\gamma \cdot\{(U, \pi)\}=\{\gamma \cdot(U, \pi)\} .
$$

3.1. Proposition. Let $h$ be the element of $H^{2}(L, M)$ determined by $(U, \pi)$. Then the element of $H^{2}(L, M)$ determined by $\gamma \cdot\{(U, \pi)\}$ is $\gamma \cdot h$.

Proof. Let $\alpha$ be a linear map of $L$ into $U$ such that $\pi \alpha(x)=x$ for all $x$ in $L$. The element $f$ of $C^{2}(L, M)$ such that

$$
f\left(x_{1}, x_{2}\right)=\left[\alpha\left(x_{1}\right), \alpha\left(x_{2}\right)\right]-\alpha\left(\left[x_{1}, x_{2}\right]\right)
$$

is in $Z^{2}(L, M)$ and represents $h$. Define a linear map $\hat{\alpha}: L \rightarrow U_{\gamma} / M_{\gamma}$ by

$$
\hat{\alpha}(x)=\langle\alpha(x), \alpha([\gamma, x])\rangle+M_{\gamma}
$$

Then

$$
\left[\hat{\alpha}\left(x_{1}\right), \hat{\alpha}\left(x_{2}\right)\right]-\hat{\alpha}\left[x_{1}, x_{2}\right]=(\gamma \cdot f)\left(x_{1}, x_{2}\right) \quad \text { for all } x_{1}, x_{2} \text { in } L .
$$

3.2. Corollary. The operation of $G$ on $\operatorname{Ext}(M, L)$ induces the structure of a $G$-module on $\operatorname{Ext}(M, L)$ and the vector space isomorphism of $\operatorname{Ext}(M, L)$ onto $H^{2}(L, M)$ becomes a $G$-isomorphism.

Next consider $\operatorname{Ext}(M, G)$. If $\{(U, \pi)\}$ is $\operatorname{in} \operatorname{Ext}(M, G)$ put

$$
\rho\{(U, \pi)\}=\left\{\left(\pi^{-1}(L), \bar{\pi}\right)\right\}
$$


in $\operatorname{Ext}(M, L)$ where $\bar{\pi}$ is the restriction of $\pi$ to $\pi^{-1}(L) . \rho$ is a homomorphism of $\operatorname{Ext}(M, G)$ into $\operatorname{Ext}[M, L]$ and it is evident that the cohomology class in $H^{2}(L, M)$ belonging to $\rho\{(U, \pi)\}$ is $\rho_{2}(h)$ where $g$ is the cohomology class in $H^{2}(G, M)$ belonging to $\{(U, \pi)\}$ and $\rho_{2}$ is the restriction homomorphism.

3.3. Proposition. $\rho$ Ext $[M, G] \subseteq \operatorname{Ext}[M, L]^{G}$.

Proof. $\bar{U}=\pi^{-1}(L)$ is an ideal in $U$. Let $\bar{\pi}=\pi \mid \bar{u}$ then $\gamma \cdot(\bar{U}, \bar{\pi})$ is equivalent to the split extension $(L, M)$ of $M$ by $L$. In fact, the map

$$
\begin{aligned}
\left\langle\bar{u}_{1}, \bar{u}_{2}\right\rangle+M_{\gamma} \rightarrow & \left(\bar{\pi}_{(}\left(\bar{u}_{1}\right), \bar{u}_{2}-\left[\gamma^{1}, \bar{u}_{1}\right]\right) \\
& \left(\bar{u}_{i} \text { in } \bar{U}, \gamma^{\prime} \text { in } \bar{U} \text { such that } \pi\left(\gamma^{\prime}\right)=\gamma\right)
\end{aligned}
$$

of $\bar{U}_{\gamma} / M_{\gamma}$ onto $(L, M)$ is an equivalence isomorphism.

Again we have a commutative diagram:

$$
\begin{aligned}
& \operatorname{Ext}(M, G) \stackrel{\rho}{\rightarrow} \operatorname{Ext}(M, L)^{a} \\
& H^{2}(G, M) \stackrel{\rho_{1}}{\rightarrow} H^{2}(\stackrel{\uparrow}{L}, M)^{G}
\end{aligned}
$$

where $\rho$ and $\rho_{2}$ are homomorphisms into. If $G / L$ is semi-simple and if the ground field has characteristic 0 we know that $\rho_{2}$ is an isomorphism onto by 1.1 . Thus

3.5. Proposition. If $G / L$ is semi-simple and if the ground field of $G$ has characteristic $0, \rho$ is an isomorphism of $\operatorname{Ext}(M, G)$ onto $\operatorname{Ext}(M, L)^{G}$.

4. Lie algebra kernels. As before, let $G$ be a Lie algebra, $L$ an ideal of $G$ and $M$ a $G$-module. The derivations of $L$ form a Lie algebra (denoted by $D(L)$ ) and the inner derivations form an ideal of $D(L)$ denoted by $I(L)$. An $L$-kernel with nucleus $M$ is a pair $(K, \phi)$ consisting of a Lie algebra $K$ and a homomorphism $\phi$ of $L$ into $D(K) / I(K)$, $M$ is required to be the center of $K$ and $\phi$ must induce the given $L$ module structure on $M$.

Hochschild [3, p. 699 et seq.], has defined a rule of composition of $L$-kernels and a partitioning of the set of $L$-kernels with nucleus $M$ into similarity classes such that the resulting set of similarity classes of kernels, Kern $[L, M]$, becomes a vector space over the same ground field as $L$. It is not difficult to show that each element of Kern $[L, M]$ determines a unique element of $H^{3}(L, M)$ and that this correspondence is in fact a vector space isomorphism of Kern $[L, M]$ into $H^{3}(L, M)$. This isomorphism is not onto in general and again we refer to $[3]$ and $[4]$ for the details. 
Here we shall define directly an operation of $G$ on $\operatorname{Kern}[L, M]$ which is "correct" in the sense that if the similarity class $\{[K, \phi)\}$ determines $h$ in $H^{3}(L, M)$ then $\gamma \cdot\{(K, \phi)\}$ determines $\gamma \cdot h$. An immediate consequence of this (at least if the ground field has characteristic 0$)$ is that the effaceable elements of $H^{3}(L, M)$ constitute a $G$ submodule of $H^{3}(L, M)$. See [4, Theorem 5, p. 777].

In order to do this, let $(K, \phi)$ be an $L$-kernel with nucleus $M$ where $L$ is an ideal of $G$ and take $\gamma$ in $G$. Form the Lie algebra $K_{\gamma}$ of pairs $\left\langle k_{1}, k_{2}\right\rangle$ with $k_{1}, k_{2}$ in $K$ and put

$$
\left[\left\langle k_{1}, k_{2}\right\rangle,\left\langle k_{1}^{\prime}, k_{2}^{\prime}\right\rangle\right]=\left\langle\left[k_{1}, k_{1}^{\prime}\right],\left[k_{1}, k_{2}^{\prime}\right]+\left[k_{2}, k_{1}^{\prime}\right]\right\rangle \cdot
$$

$K_{\gamma}$ has center $\langle M, M\rangle$ and we define a homomorphism $t_{\gamma}:\langle M, M\rangle \rightarrow M$ such that $t_{\gamma}\left\langle m_{1}, m_{2}\right\rangle=\gamma \cdot m_{1}+m_{2}$. The kernel $T_{\gamma}$ of $t_{\gamma}$ consists of pairs $\langle m,-\gamma \cdot m\rangle$ with $m$ in $M$.

Now form $K_{\gamma} / T_{\gamma}$. The center of $K_{\gamma} / T_{\gamma}$ is $\langle M, M\rangle / T_{\gamma}$; for if $\left[\left\langle a_{1}, a_{2}\right\rangle,\left\langle k_{1}, k_{2}\right\rangle\right]$ is in $T_{\gamma}$ for all $\left\langle k_{1}, k_{2}\right\rangle$ in $K_{\gamma}$ then $\left[a_{1}, k_{1}\right]$ is in $M$ for all $k_{1}$ in $K$ and $\left[a_{1}, k_{2}\right]+\left[a_{2}, k_{1}\right]=-\gamma \cdot\left[a_{1}, k_{1}\right]$. In particular, for pairs of the form $\langle m, k\rangle$ with $m$ in $M$, we get

$$
\left[a_{1}, k\right]+\left[a_{2}, m\right]=-\gamma \cdot\left[a_{1}, m\right]=0
$$

whence $\left[a_{1}, k\right]=0$ for all $k$ in $K$ so that $a_{1}$ is in $M$. Thus since $\left[a_{1}, k_{2}\right]$ $+\left[a_{2}, k_{1}\right]=-\gamma \cdot\left[a_{1}, k_{1}\right]$ for all $\left\langle k_{1}, k_{2}\right\rangle$, we see that $\left[a_{2}, k_{1}\right]=0$ for all $k_{1}$ in $K$ i.e. $a_{2}$ is in $M$.

We define a homomorphism $\phi_{\gamma}$ of $L$ into $D\left(K_{\gamma} / T_{\gamma}\right) / I\left(K_{\gamma} / T_{\gamma}\right)$ as follows: let $\rho$ be any linear map of $L$ into $D(K)$ which is compatible with $\phi$ (i.e. the class $\bmod I(K)$ of $\rho(x)$ is $\phi(x)$ for all $x$ in $L$.) Set

$$
\rho^{*}(x)\left\{\left\langle k_{1}, k_{2}\right\rangle+T_{\gamma}\right\}=\left\langle\rho(x)\left\{k_{1}\right\}, \rho(x)\left\{k_{2}\right\}-\rho([\gamma, x])\left\{k_{1}\right\}\right\rangle+T_{\gamma} .
$$

It is easy to check that $\rho^{*}$ is a linear map of $L$ into $D\left(K_{\gamma} / T_{\gamma}\right)$ and that the class $\bmod I\left(K_{\gamma} / T_{\gamma}\right)$ of $\rho^{*}(x)$ does not depend on the choice of $\rho$ compatible with $\phi$. Now we note that if

$$
\left[\rho\left(x_{1}\right), \rho\left(x_{2}\right)\right]-\rho\left(\left[x_{1}, x_{2}\right]\right)=D_{\tau\left(x_{1}, x_{2}\right)} \quad \text { in } I(K) \text { for } x_{1}, x_{2} \text { in } L,
$$

then

$$
\left[\rho^{*}\left(x_{1}\right), \rho^{*}\left(x_{2}\right)\right]-\rho^{*}\left(\left[x_{1}, x_{2}\right]\right)=D_{\tau *\left(x_{1}, x_{2}\right)}
$$

where $\tau^{*}\left(x_{1}, x_{2}\right)=\left\langle\tau\left(x_{1}, x_{2}\right),-\tau\left(\left[\gamma, x_{1}\right], x_{2}\right)-\tau\left(x_{1},\left[\gamma, x_{2}\right]\right)\right\rangle+T_{\gamma}$. Now put $\phi_{\gamma}(x)=\phi^{*}(x)+I\left(K_{\gamma} / T_{\gamma}\right)$. One sees immediately that $\phi_{\gamma}$ induces the given $L$-module structure on the center $\langle M, M\rangle / T_{\gamma}$ and that $t_{\gamma}$ induces an $L$-isomorphism of $\langle M, M\rangle / T_{\gamma}$ onto $M$ so that the pair $\left(K_{\gamma} / T_{\gamma}, \phi_{\gamma}\right)$ is an $L$-kernel with nucleus $M$ which we denote by $\gamma \cdot(K, \phi)$. 
The function $\tau: L \times L \rightarrow K$ is called a hindrance of $(K, \phi)$ and its (formal) coboundary $\delta \tau$ is an element of $Z^{3}(L, M)$ called a primary deviation. The cohomology class of $\delta \tau$ (called the obstruction of $(K, \phi))$ is independent of the choice of $\rho$ compatible with $\phi$ and of the choice of $\tau$ such that $\left[\rho\left(x_{1}\right), \rho\left(x_{2}\right)\right]-\rho\left(\left[x_{1}, x_{2}\right]\right)=D_{\tau\left(x_{1}, x_{2}\right)}$.

In this terminology, $\tau^{*}\left(x_{1}, x_{2}\right)$ is a hindrance of $\gamma \cdot(K, \phi)$ and a straightforward computation shows that

$$
\delta \tau^{*}=\gamma \cdot \delta \tau .
$$

Since two $L$-kernels have the same obstruction if and only if they are similar, we have

4.1. Proposition. The map $\{(K, \phi)\} \rightarrow\{\gamma \cdot(K, \phi)\}$ (where braces indicate similarity class) induces the structure of a G-module on Kern $[L, M]$ and further, if $h$ is the obstruction of $\{(K, \phi)\}$, then $\gamma \cdot h$ is the obstruction of $\{\gamma \cdot(K, \phi)\}$.

As before we define a map $\rho$ of Kern $[G, M]$ into Kern $[L, M]$ by setting $\rho\{(\hat{K}, \hat{\phi})\}=\left\{\left(\hat{K},\left.\widehat{\phi}\right|_{L}\right)\right\}$ where $\left.\widehat{\phi}\right|_{L}$ is the restriction of $\hat{\phi}$ to $L$. It is evident that if $h$ is the obstruction of $\{(\hat{K}, \hat{\phi})\}$ then $\rho_{3}(h)$ is the obstruction of $\left\{\left(\hat{K},\left.\hat{\phi}\right|_{L}\right)\right.$ where $\rho_{3}$ is the restriction map of $H^{3}(G, M)$ into $H^{3}(L, M)$. Again we have a commutative diagram:

$$
\begin{array}{ccc}
H^{3}(G, M) & \rightarrow & H^{3}(L, M)^{G} \\
\downarrow & \downarrow \\
\operatorname{Kern}[G, M] & \rightarrow \operatorname{Kern}[L, M]^{G}
\end{array}
$$

Appendix

For details concerning the following discussion the reader is referred to [5].

\section{A. Group enlargements.}

DEFINITION. Let $G$ be an arbitrary group (written multiplicatively) and $P, Q$ groups on which $G$ operates from the left. In this section, $P$ is assumed commutative but $Q$ need not be. A $G$-enlargement of $P$ by $Q$ is a pair $(E, \phi)$ such that

(i) $G$ operates on $E$ from the left,

(ii) $P$ is a subgroup of $E$ and a direct summand of $E$ (as a group),

(iii) $\phi$ is a $G$-homomorphism of $E$ onto $Q$ with kernel $P$.

Two $G$-enlargements $\left(E_{1}, \phi_{1}\right),\left(E_{2}, \phi_{2}\right)$ are called equivalent if there exists a $G$-isomorphism $\tau: E_{1} \rightarrow E_{2}$ such that $\tau(p)=p$ for all $p$ in $P$ and $\phi_{2} \tau=\phi_{1}$.

Eilenberg gives a definition of multiplication of equivalence classes 
of $G$-enlargements so that these classes form an abelian group denoted by $\operatorname{Enl}(G, Q, P)$. If $\theta$ is an element of $\operatorname{Hom}(Q, P)$ and if one puts $(\sigma \cdot \theta)\{q\}=\sigma \cdot \theta\left(\sigma^{-1} q\right)$ for all $\sigma$ in $G$ and $q$ in $Q$ then $\operatorname{Hom}(Q, P)$ becomes a $G$-module and Eilenberg then showed that ENL $(G, Q, P)$ $\cong H^{1}(G, \operatorname{Hom}(Q, P))$.

Now suppose that $G$ is a normal subgroup of a group $\Gamma$ and that $\Gamma$ also operates on $P$ and $Q$. Let $(E, \phi)$ denote a $G$-enlargement of $P$ by $Q$. Take $\gamma$ in $\Gamma$ and let $E_{\gamma}$ be the subgroup of $E \times E$ consisting of pairs $\left(e_{1}, e_{2}\right)$ such that $\phi\left(e_{1}\right)=\boldsymbol{\gamma} \cdot \boldsymbol{\phi}\left(e_{2}\right)$. Make $E_{\gamma}$ into a $G$-module such that $\sigma \cdot\left(e_{1}, e_{2}\right)=\left(\sigma \cdot e_{1}, \sigma^{\gamma} e_{2}\right)$ where $\sigma^{\gamma}=\gamma^{-1} \sigma \gamma$ for all $\sigma$ in $G$. Let $\phi_{\gamma}^{*}$ denote the $G$-homomorphism $\left(e_{1}, e_{2}\right) \rightarrow \phi\left(e_{1}\right)$ of $E_{\gamma}$ onto $Q$. The kernel of $\phi_{\gamma}^{*}$ is $(P, P)$ and the map $\alpha_{\gamma}:(P, P) \rightarrow P$ such that $\alpha_{\gamma}\left(p_{1}, p_{2}\right)=\gamma \cdot p_{2}$ is a $G$-homomorphism of $(P, P)$ whose kernel $\operatorname{ker}\left(\alpha_{\gamma}\right)$ consists of pairs $(p, 1)$ where 1 is the identity of $P . \phi_{\gamma}^{*}$ induces a $G$-homomorphism $\phi_{\gamma}$ of $E_{\gamma} / \operatorname{ker}\left(\alpha_{\gamma}\right)$ onto $Q$ so that the pair $\left(E_{\gamma} / \operatorname{ker}\left(\alpha_{\gamma}\right), \phi_{\gamma}\right)$ is a $G$-enlargement of $P$ by $Q$ which we denote by $\gamma \cdot(E, \phi)$. It is not difficult to show that if $(E, \phi)$ is equivalent to $\left(E^{\prime}, \phi^{\prime}\right)$ then $\gamma \cdot(E, \phi)$ is equivalent to $\gamma \cdot\left(E^{\prime}, \phi^{\prime}\right)$ and that this operation induces the structure of a $\Gamma$-module on $\operatorname{Enl}(G, Q, P)$. Further one can check that this structure is compatible with the $\Gamma$-module structure on $H^{1}(G$, Hom $(Q, P))$ so that the natural isomorphism of $\operatorname{Enl}(G, Q, P)$ onto $H^{1}(G$, Hom $(Q, P))$ becomes a $\Gamma$-isomorphism.

B. Group extensions. If $M$ is a subgroup of $U$ and if the sequence $0 \rightarrow M \rightarrow U \rightarrow \pi G \rightarrow 0$ is exact then we say that the pair $(U, \pi)$ is an extension of $G$ by $M$. If $M$ is abelian, this situation makes $M$ into a $G$-module. Two such extensions $\left(U_{1}, \pi_{1}\right)$ and $\left(U_{2}, \pi_{2}\right)$ of $G$ by $M$ are called equivalent if there exists an isomorphism $\tau: U_{1} \rightarrow U_{2}$ such that $\tau(m)=m$ for all $m$ in $M$ and such that $\pi_{1}=\pi_{2} \tau$. The equivalence classes of such extensions form an abelian group $A(G, M)$ which is isomorphic with $H^{2}(G, M)$ see [5].

Now suppose that $G$ is a normal subgroup of a group $\Gamma$ and that $M$ is also a $\Gamma$-module. Let $(U, \pi)$ be an extension of $G$ by $M$ and take $\gamma$ in $\Gamma$. Form $U_{\gamma}=\left\{\left\langle u_{1}, u_{2}\right\rangle \mid u_{i}\right.$ in $\left.U, \pi\left(u_{1}\right)=\pi\left(u_{2}\right)^{\gamma}\right\}$ with $\left\langle u_{1}, u_{2}\right\rangle$ $\left\langle u_{1}^{\prime}, u_{2}^{\prime}\right\rangle=\left\langle u_{1} u_{1}^{\prime}, u_{2} u_{2}^{\prime}\right\rangle$. Set $\pi_{\gamma}\left\langle u_{1}, u_{2}\right\rangle=\pi\left(u_{1}\right)$ and note that the kernel of $\pi_{\gamma}$ is $\langle M, M\rangle$. Define a homomorphism $\omega_{\gamma}:\langle M, M\rangle \rightarrow M$ by $\omega_{\gamma}\left\langle m_{1}, m_{2}\right\rangle=\gamma \cdot m_{2}$ for all $\left\langle m_{1}, m_{2}\right\rangle$ in $M . \omega_{\gamma}$ is a $G$-homomorphism of $\langle M, M\rangle$ onto $M$ and the kernel of $\omega_{\gamma} \equiv\langle M, 0\rangle$ is a normal subgroup of $U_{\gamma} \cdot \pi_{\gamma}$ induces a homomorphism (still denoted by $\pi_{\gamma}$ ) of $U_{\gamma} /\langle M, 0\rangle$ onto $M$ and we put

$$
\gamma \cdot(U, \pi)=\left(U_{\gamma} /\langle M, 0\rangle, \pi_{\gamma}\right) .
$$

One can check that if $(U, \pi)$ is equivalent to $\left(U_{1}, \pi_{1}\right)$ then $\gamma \cdot(U, \pi)$ 
is equivalent to $\gamma \cdot\left(U_{1}, \pi_{1}\right)$ and that this operation induces the structure of a $\Gamma$-module on $A(G, M)$. Further, if $h$ is the element of $H^{2}(G, M)$ belonging to $(U, \pi)$ then $\gamma \cdot h$ belongs to $\gamma \cdot(U, \pi)$ so that the isomorphism of $A(G, M)$ onto $H^{2}(G, M)$ becomes a $\Gamma$-isomorphism.

C. Group kernels. A $G$-kernel is a pair $(K, \theta)$ where $\theta$ is a homomorphism: $G \rightarrow A(K) / I(K)$ (the outer automorphisms of $K$ ). If $M$ is the center of $K$, then $\theta$ makes $M$ into a $G$-module called the nucleus of $(K, \theta)$. Eilenberg-MacLane [5, p. 12] have given a rule of composition of $G$-kernels with fixed nucleus $M$ and a partitioning of the set of $G$-kernels with nucleus $M$ into similarity classes such that the resulting set of similarity classes $\mathscr{K}(G, M)$ becomes an abelian group. They then show that $\Re(G, M)$ is isomorphic to $H^{3}(G, M)$.

Now suppose that $G$ is a normal subgroup of a group $\Gamma$ and that $M$ is also a $\Gamma$-module. Let $(K, \theta)$ be a $G$-kernel with nucleus $M$ and take $\gamma$ in $\Gamma$. As a group, put $K_{\gamma}=K$ and put $\theta_{\gamma}(\sigma)=\theta\left(\gamma^{-1} \sigma \gamma\right)$ for all $\sigma$ in $G$. The center $M_{\gamma}$ of $K_{\gamma}$ is still $M$ (as a group) but now the operation of $G$ on $M_{\gamma}$ is given by

$$
\sigma * m=\gamma^{-1} \sigma \gamma \cdot m \quad \text { for all } \sigma \text { in } G, m \text { in } M .
$$

The map $m \rightarrow \gamma \cdot m$ is a $G$-isomorphism of $M_{\gamma}$ onto $M$ which we use to identify $M_{\gamma}$ with $M$ as a $G$-module. Then the pair $\left(K_{\gamma}, \theta_{\gamma}\right)$ is a $G$ kernel with nucleus $M$ which we denote by $\gamma \cdot(K, \theta)$. One can check that this operation induces the structure of a $\Gamma$-module on $\mathscr{K}(G, M)$ and sees that if $h$ is the element of $H^{3}(G, M)$ belonging to $(K, \theta)$ then $\gamma \cdot h$ belongs to $\gamma \cdot(K, \theta)$ so that the isomorphism of $\mathscr{K}(G, M)$ with $H^{3}(G, M)$ becomes a $\Gamma$-isomorphism.

\section{BIBLIOGRAPHY}

1. G. Hochschild and J.-P. Serre, Cohomology of Lie algebras, Ann. of Math. vol. 57 (1953) pp. 591-603.

2. G. Hochschild, Cohomology of restricted Lie algebras, Amer. J. Math. vol. 66 (1954) pp. 555-580.

3. - Lie Algebra kernels and cohomology, Amer. J. Math. vol. 66 (1954) pp. $698-716$.

4. - Cohomology classes of finite type and finite dimensional kernels for Lie algebras, Amer. J. Math. vol. 66 (1954) pp. 763-778.

5. Samuel Eilenberg, Topological methods in abstract algebra, Bull. Amer. Math. Soc. vol. 55 (1949) pp. 3-37.

SyRACUSE UNiversity AND

UNIVERSITY oF PitTsBURGh 